Canad. Math. Bull. Vol. 21 (2), 1978

\title{
HOW FIELDS CAN HAVE A PRODUCT
}

\author{
BY \\ FRANK ZORZITTO
}

Let $k$ be a field. Two field extensions $E, F$ of $k$ are said to have a product in the category of field extensions of $k$ (see e.g. [1, p. 30]) if and only if there exist a field extension $P$ of $k$ and two $k$-isomorphisms $P \rightarrow E, P \rightarrow F$ satisfying the following universal property. For any field extension $K$ of $k$ and any pair of $k$-isomorphisms $K \rightarrow E, K \rightarrow F$, there exists a unique $k$-isomorphism $K \rightarrow P$ such that the diagrams below commute.
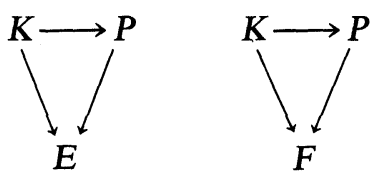

For many common categories, such as groups or rings, the object having the above universal property is constructed by taking the usual cartesian product. While the cartesian product of two fields is never a field, it is sometimes possible for two fields to have a produci in the categorical sense. Henceforth, the term product shall mean product in the category of field extensions of $k$.

THEOREM. Two field extensions $E, F$ of $k$ have a product if and only if

(i) for any $x$ in $E$ there exists at most one $k$-conjugate $x_{1}$ in $F$, and

(ii) for any $x, y$ in $E$ with respective $k$-conjugates $x_{1}, y_{1}$ in $F$ and any polynomial $p(X, Y)$ in $k[X, Y]$, the condition $p(x, y)=0$ is equivalent to $p\left(x_{1}, y_{1}\right)=0$.

Two elements are called $k$-conjugates if they both satisfy the same irreducible polynomial over $k$ or if they are both transcendental.

The necessity of these conditions is left to the reader to prove. Here is a proof of their sufficiency. Let $P$ be the set of those $x$ in $E$ which have a $k$-conjugate in $F$. Every $z$ in $P$ is algebraic over $k$. Otherwise, if $z$ in $P$ with conjugate $z_{1}$ in $F$ were transcenderital, then $z$ would also have the transcendental element $1+z_{1}$ in $F$ as a conjugate, contrary to condition (i). Let $x, y$ in $P$ have conjugates $x_{1}, y_{1}$ respectively in $F$. The conjugate in $F$ of the algebraic element $x+y$ in $E$ is $x_{1}+y_{1}$. This is clear because condition (ii) implies that $p(X)$ in $k[X]$ is the minimal polynomial of $x_{1}+y_{1}$ given that $p(X)$ is the minimal polynomial of $x+y$. Thus $x+y \in P$. A similar argument proves that

Received by the editors July 8, 1976 . 
$x y,-x, x^{-1} \in P$, implying that $P$ is a field. Let $P \rightarrow E$ be the inclusion mapping, and let $P \rightarrow F$ be the mapping that sends $z$ in $P$ to its unique conjugate $z_{1}$ in $F$. These mappings are then the $k$-isomorphisms which establish $P$ as the product of $E$ and $F$.

Here are some examples of products of fields.

If $E, F$ are in the algebraic closure of $k$ and linearly disjoint (see e.g. [1, p. 261]) over $k$, their product exists and equals $k$. To see this let $x$ in $E$ have $k$-conjugate $x_{1}$ in $F$. It follows that $x \in k$ and $x=x_{1}$, whereby conditions (i) and (ii) are met. Indeed, let $p(X)=X^{n}+a_{n-1} X^{n-1}+\cdots+a_{1} X+a_{0}$ in $k[X]$ be the minimal polynomial of $x$ and $x_{1}$. Thus $p(x)-p\left(x_{1}\right)=$ $\left(x^{n}-x_{1}^{n}\right)+\cdots+a_{1}\left(x-x_{1}\right)=0$. If $x$ differed from $x_{1}$, we could divide by $x-x_{1}$ to get a $k$-linear dependence relation among the elements $\left\{x^{i} x_{1}^{j}: i, j=\right.$ $0, \ldots, n-1\}$. The linear disjointness of $E$ and $F$ would then imply a $k$-linear dependence relation on either the elements $\left\{1, x, \ldots, x^{n-1}\right\}$ or the elements $\left\{1, x_{1}, \ldots, x_{1}^{n-1}\right\}$, in contradiction to the minimality of $p(X)$. Thus $x=x_{1}$; and since $E \cap F=k$ by linear disjointness, then $x \in k$.

For a second example, let $p, q$ be odd positive primes, and consider the field extension $Q(\sqrt[p]{q} q)$ of the rationals $Q$, where $\sqrt[p]{q}$ is the real $p$ th root of $q$. The product of $Q(\sqrt[p]{q} q)$ with itself exists in the category of field extensions of $Q$, since conditions (i) and (ii) hold for $E=Q(\sqrt[p]{q}), F=Q(\sqrt[p]{q} q)$.

This last example raises some field theoretical questions. The class of field extensions of $Q$, which do have a product with themselves, may be considered anti-normal as they are characterized by an utter lack of conjugates, according to condition (i). Do there exist such infinite dimensional anti-normal extensions of $Q$ ? Being an anti-normal extension of $Q$ is an inductive property. Thus there are maximal anti-normal extensions in the algebraic closure of $Q$. Are these isomorphic? In what manner can they be classified?

I would like to thank the referee for pointing out that the above condition (i) follows from condition (ii) with $p(X, Y)=X-Y$.

\section{REFERENCE}

1. Serge Lang, Algebra, (Addison-Wesley, 1965).

Dept. of Math.

UNIVERSITY OF WATERLOO,

WATERLOO, ONTARIO,

CANADA N2L 3G1 